This paper is published as:

Joseph, Shannon A, Adams, Barry J, McCabe, BY, 2010, “A Methodology for Bayesian Belief Network Development to Facilitate Compliance with Water Quality Regulations", Journal of Infrastructure Systems, 16(1):58-65

\title{
A Methodology for Bayesian Belief Network Development to Facilitate Compliance with Water Quality Regulations
}

\author{
Shannon A. Joseph ${ }^{1}$ and Barry J. Adams M.ASCE${ }^{2}$, Brenda McCabe ${ }^{3}$
}

\begin{abstract}
Limited resources and drinking water quality requirements pose significant challenges to those managing small and rural drinking water distribution systems (WDSs). Real-time monitoring technologies could support regulatory compliance, if shortcomings such as false readings and data corruption could be overcome. Bayesian Belief Networks (BBNs) are proposed as a means to mitigate technological shortcomings and increase certainty about the state of a given WDS. This paper describes a methodology for the development of BBNs that integrates known system characteristics with real-time monitoring technologies to support the water quality compliance of small or rural WDSs. Expert judgement was used both in the development of the structure of the BBN and in quantifying the required probability relationships. The results of a case study application of this methodology suggest that it is useful in developing a BBN to support decision making for a WDS with limited use of realtime monitoring technology.
\end{abstract}

\section{Subject Headings}

Bayesian Analysis, Decision support systems, Expert Systems, Public Safety, Drinking Water, Remote Sensing

\section{Introduction}

Ensuring the regulatory compliance of drinking water distribution systems (WDSs) is an expensive enterprise for any utility. Frequent sampling, maintenance and associated record keeping require substantial human and financial resources that are often limited in the context of small or rural water distribution systems. It is therefore not unexpected that the major drinking water quality failure cases of the developed world, in which regulatory standards are not met and there are serious health consequences, occur in small or rural communities (Hrudley and Hrudley 2004). The most serious Canadian drinking water related health crises in recent history occurred between 2000 and 2005 in the rural communities of Walkerton, Ontario, North Battleford

\footnotetext{
${ }^{1}$ M.A.Sc. Graduate, Department of Civil Engineering, University of Toronto, 35 St. George Street, Toronto, M5S 1A4 Canada, E-mail - shannon.joseph@utoronto.ca

${ }^{2}$ Professor, Department of Civil Engineering, University of Toronto, 35 St. George Street, Toronto, M5S 1A4 Canada, E-mail-adams@ecf.utoronto.ca

${ }^{3}$ Professor and Chair, Department of Civil Engineering, University of Toronto, 35 St. George Street, Toronto, M5S 1A4 Canada, E-mail - brenda.mccabe@utoronto.ca
} 
Saskatchewan and the Kashechewan First Nations reserve (Hrudley and Hrudley 2004, Strauss 2005). These and other recent outbreaks have signalled to the developed world that small utilities require more support than they have received in the past in order to ensure drinking water safety.

Information technologies such as sensors and Supervisory Control and Data Acquisition (SCADA) systems could provide this support; they make it possible to acquire water quality data at a high frequency for multiple areas at a time in an often widely distributed system. However, power supply, security, false readings and data transmission challenges are elements that can significantly compromise the benefits of investments in real-time technology, particularly for small utilities (Bristow and Brumbelow 2006). Furthermore, the limited resources of small utilities constrain the number and types of sensors that can be purchased for WDS monitoring thus prohibiting the use of sampling density as a safeguard against false readings and corrupted data. A methodology is required to support the technology, such that the benefits of remote monitoring can be realized with an acceptable degree of confidence about the reliability of the information.

Bayesian Belief Networks (BBNs) have been identified as a tool to support the use of realtime technology in water distribution system monitoring (Dawsey et al. 2006). BBNs are particularly appropriate for water quality uncertainty in WDSs because they can be used to integrate observable and non-observable phenomena into a probability-based framework. This framework allows for the calculation of the probability of non-observable phenomena based on the known state of observable phenomena, such as a sensor reading. Dawsey and co-authors were first to use BBNs to address the problem of drinking water safety with real-time monitoring in a water distribution system. The present work advances their initial application by developing and testing a methodology for the construction of a BBN for water quality management in the context of small WDSs.

Some of the unique features of small utilities include limited availability of computer models or drawings of the system, historical hydraulic and water quality data with low temporal and spatial density and a small number of system experts. This methodology investigates in particular the use of expert judgment in the development of a BBN as a way to overcome limited data for small water systems. This element makes it potentially generalizable to BBN development for other WDSs for which there is limited data.

There are numerous challenges associated with the use of expert probability assessment for decision making. Biases and the use of heuristics, as well as limitations in the number of conditions an expert can consider, are just a few of the key considerations in using this method of probability generation (Tversky 1974, Meyer and Booker 2001). To overcome these challenges, questions for probability elicitation must be carefully developed and experts must be carefully selected and trained for the task (Renooij 2001, Keeney and Von Winterfeldt 1991, Meyer and Booker 2001). This is particularly important in addressing the gap between the experts' knowledge in their field and their familiarity with the formulation of this knowledge as a probability values. Decisions about the number of experts to engage are equally important, although in the context of small water utilities there may be a limited number of experts. A review of the literature suggests that three to five experts represent an acceptable number beyond which there is increased redundancy in the data for each additional expert (Clemen and Winkler 1999, O'Leary 1998). While expert data is often not calibrated (unbiased), each expert's set of probability assessments for a given BBN conveys his or her experience with the system and complements the knowledge of other experts with different experiences (Stiber et al. 2004, Meyer and Booker 2001). The authors acknowledge that there are limitations associated with the use of expert probability assessment but employed available measures to minimize their impacts. 
The methodology was developed and tested on a case study system in rural Canada. This paper describes the process of BBN structure development and probability elicitation. It also presents the processes used to test the characteristics of the BBN and the sensitivity of each expert data set to error. Since more than one expert was relied upon to generate the needed probabilities for the model, the procedure used to combine the expert data is described in detail. Finally, the combined expert BBN was tested using historical data for which the causes of various water quality anomalies were known. The performance of the BBN as a decision making tool for small or rural WDSs, and its implications for the use of the methodology, are discussed.

\section{Bayesian Belief Network Theory}

BBNs are directed acyclic graphs composed of nodes and arrows. The nodes represent variables (ideally, mutually exclusive and collectively exhaustive); arrows, represent probabilistic dependence relationships between the variables. Arrows lead from parent nodes to one or more child nodes; nodes without parents (a priori) are called orphans (Jensen 1996). There are two categories of nodes in BBNs: 1) hypothesis nodes, representing phenomena that cannot be observed directly but are of interest to the BBN user and 2) input nodes, representing observable phenomena (Jensen 1996). The influence of a parent node on the child is quantified by conditional probabilities (Coupé and Van der Gaag 2002). The number of probabilities required to complete a node's probability table is expressed by Equation 1 (Tang 2005):

$$
N P=(m-1) \prod_{i=1}^{k} n_{i}
$$

where $N P=$ number of probabilities; $m=$ number of possible, mutually exclusive, states of the child node; $n_{i}=$ number of possible, mutually exclusive, states of parent node " $i$ " where $i$ ranges from 1 to $k$. If $i=0$, then this term drops out (as opposed to being zero). The probabilities can be obtained from frequency analysis of data, expert assessment, or a combination of both.

Once all conditional probability relationships are quantified within the $\mathrm{BBN}$, it is possible to calculate the posterior probability of hypothesis nodes given the state of one or more input nodes (Dawsey et al. 2006). Bayes' theorem, the basis by which probabilities are propagated throughout the network (Pearl 1988), is demonstrated in Equations 2 through 4.

$$
P(A \cap B)=P\langle A \mid B\rangle P(B)
$$

Because $P(A \cap B)=P(B \cap A)$ it follows that,

$$
P(A \cap B)=P\langle B \mid A\rangle P(A)
$$

Bayes rule takes advantage of this property and yields

$$
P\langle A \mid B\rangle=\frac{P\langle B \mid A\rangle P(A)}{P(B)}
$$

where $P(A)=$ prior probability of event $A, P(B)=$ prior probability of event $B, P(A \cap B)=$ probability of the intersection of $A$ and $B$, and $P\langle A \mid B\rangle=$ posterior probability of $A$ given $B$. Therefore, as a simple example, having observed the state of $B$ and knowing the probability of $B$ given $A$, as well as the prior probability of $A$, one can generate the updated or posterior probability for the state of hypothesis node $A$ given $B$. Therefore given a positive sensor observation, $P(B)=$ 1, the posterior probability of contamination $P\langle A \mid B\rangle$, will be greater than the prior probability of contamination $P(A)$. These probability relationships allow Bayesian Belief Networks to be flexible in handling different types of data about which there is uncertainty, and the symmetry of Bayes' 
Theorem allow probabilities to be propagated throughout the network without regard to the position of the input nodes.

Finally, any given Bayesian Network may be described completely using the chain rule which states (Jensen 1996)

$$
P(U)=\prod_{i} P\left(\left\langle A_{i} \mid p a\left(A_{i}\right)\right\rangle\right)
$$

where $U=$ the set of variables $A_{1}$ to $A_{m}$ in a Bayesian Network, $P(U)=P\left(A_{1}, \ldots, A_{m}\right)$ and $p a(A i)=$ parent set of variable $A_{i}$.

For more information on BBNs and their properties, see Pearl (1988) or Jensen (1996).

\section{Development of BBN Node Structure}

A BBN was developed in a case study of a potable water distribution system in the Canadian province of Saskatchewan. The system delivers drinking water to 5 small customer towns, as well as 28 direct connections, representing a total population of approximately 13,000 . The system, shown in Figure 1, has an average daily capacity of 700,000 gallons/day and consists of 7 principal pipe sections carrying uni-directional flow from the entrance of the system. The use of hard start pumps results in frequent moderate pressure transients whose water quality impacts are unknown. At the time of this research, the real-time instrumentation on the system consisted of a chlorine sensor and a pressure sensor at the main pumping station. Total chlorine residual and pressure was measured once daily at the delivery points of the five towns, using a portable chlorine meter and a pressure gage.

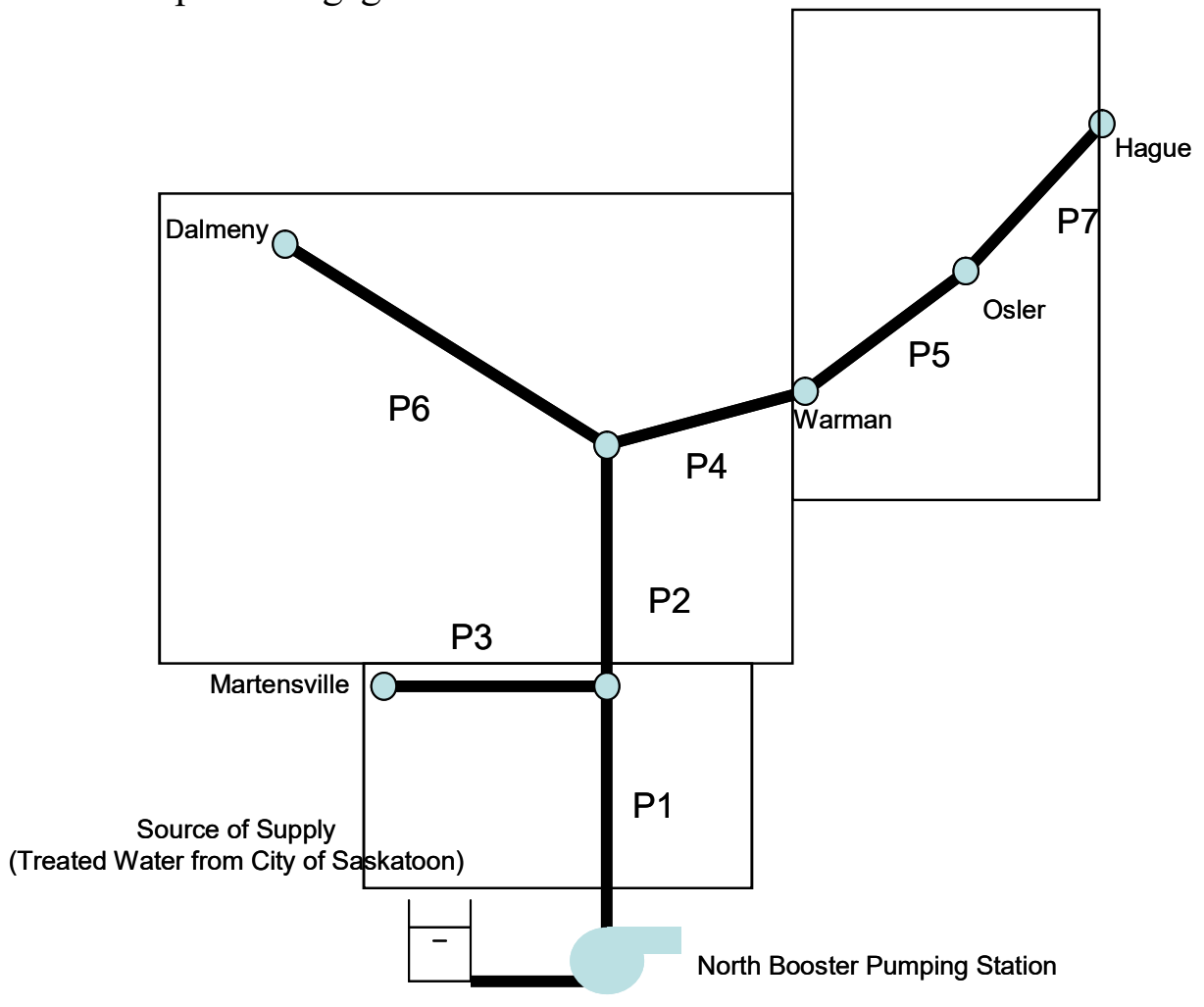

Fig. 1. Case study potable water transmission system 
The development of the structure of the BBN shown in Figure 2 consisted of four elements: 1) determining which pipe sections to group and consider together, 2) selecting which water quality risk events were most important to include, 3 ) determining which phenomena could be measured and included as indicators of non-observable events, and finally, 4) determining which additional hypothetical sensors were needed to evaluate the potential benefits of real time monitoring. Expert judgments from a general expert in WDSs from the University of Toronto, Expert D, and the case study system operator Expert C, were used at different stages of the model development. The practical objective of the BBN was to reduce uncertainty associated with the "Contamination" hypothesis nodes and the "Exposure Risk" hypothesis nodes shown in Figure 2.

The choice to divide the system into sections, prior to inlet, P1P3, P2P4P6 and P5P7 (shown in Fig. 1) was made by the authors based on drawings of the system and probable hydraulic characteristics. Subsequent discussion with the experts following the probability elicitation step of this research revealed that it may have been inappropriate to group P2 with P4 and P6 because of the presence of a pressure reducing valve at the end of P2. However, the experts asserted that this grouping was still acceptable due to other characteristics that could impact water quality including pipe material and numbers of potentially flooded valve chambers/manholes.

The primary risk conditions captured by the BBN included upstream contamination and intrusions through flooded manholes during the frequent moderate transient events. These were identified through the literature review; Robertson et al. (2003) and Lindley et al. (2002) describe water quality risks for WDSs. The variables (Exposure Risk 1, 2 and 3) were based on work that attempts to characterize the health risks posed by intrusions due to pressure transients (McInnis 2004). Nodes representing intrusion events have two parents; one representing the vulnerability of the pipe section and the other representing adverse pressure in the pipe section. Nodes representing contamination in a given area also have two parents, upstream contamination and intrusion. Expert $\mathrm{C}$ who was consulted on the risk conditions for the case study system felt these were exhaustive in the absence of foul play. Another contribution of Expert $\mathrm{C}$ was the recommendation of the inclusion of a node representing water temperature as a parent of the nodes representing water quality sensors. Contrary to the assumption of the authors that residence time was an important variable, Expert $\mathrm{C}$ asserted that water temperatures over $10 \mathrm{C}$ had a more significant impact on the stability of the chlorine residual in the summer months and overall. As a result, a single water temperature node replaced multiple residence time nodes as parents of the total chlorine sensors nodes in the BBN.

The unobservable water system events represented in the BBN are related to total chlorine, adverse pressure and the duration of a failure event. Total chlorine residual was selected as an appropriate indicator parameter of water quality failure events because it is very reactive. Six of the BBN nodes represent total chlorine monitoring for different areas in the system. A node representing the real-time pressure transducer at the entrance of the system as well as nodes at all the towns representing daily gauge pressure were included to capture the detection of "adverse pressure" events (see Figure 2). Probabilities were collected for each of these monitoring points under both actual monitoring conditions and assuming they were all real-time sensors. Under the real-time sensor scenario, an additional node representing a hypothetical real-time pressure sensor (Dalmeny Booster Pressure Transducer) is included on pipe section P6.

The final case study network consists of 36 nodes, 17 of which are hypothesis nodes, identified as ovals in Figure 2. It should be noted that the nodes Operational Change and Water Temperature can sometimes act as input nodes. 


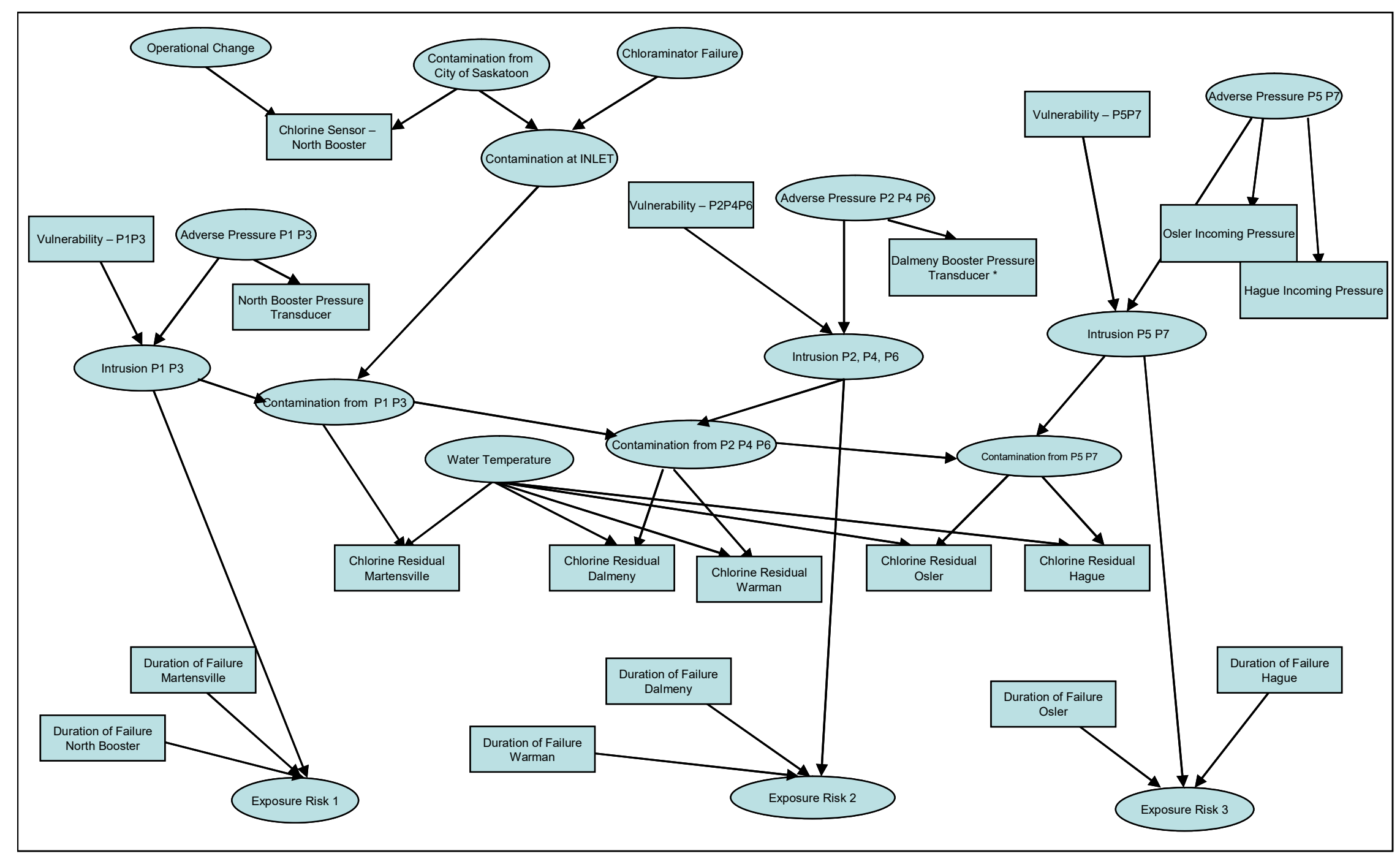

Fig. 2. Bayesian belief network structure for case study water distribution system 
At the time of probability elicitation, further refinements were made to the BBN model structure. For example, the node state definitions of the Duration of Failure nodes were changed from "one or less than one failure reading," and "two or more consecutive failure readings" to "less than one 15 minute average" and "one or more 15 minute averages," to conform more closely to the experience of the experts.

The number of conditional probabilities required to define a node increases exponentially as the number of parent nodes increases (Tang 2005). Therefore, during the development of the model structure, the maximum number of parent nodes for any variable was limited to three by determining which phenomena were most important to represent. This led to a maximum of 8 conditional probabilities to be considered by any expert. When asked, the experts who participated in the probability elicitation asserted that three parent conditions were the most they could consider simultaneously. This is consistent with the literature which identifies 7 probabilities simultaneously as an upper maximum after which the elicitation process yields less precise judgments (Meyer and Booker 2001). A draw back of this constraint on the BBN structure was exclusion of information that could have further reduced uncertainty regarding the water quality in the system. For example, an additional input node representing "low chlorine measurement in the preceding time interval" could have been included as a child of the "contamination" nodes and a parent of the "total chlorine residual" nodes. Incorporation of this time related node could have reduced uncertainty as to whether a reading was false; for every additional consistent reading, the probability that the reading is false would decrease. With the exception of time related data, additional information, such as the probability of failure of backflow protection, could be expected to be integrated into the experts' assessments of other probabilities (ie. intrusion or contamination) making the impact of their exclusion minor.

\section{Probability Elicitation}

The BBN required probability assessments for hydraulic and water quality events in areas of the WDS for which the historical data had low temporal resolution, particularly for locations that were distant from the system inlet. It was felt that expert subjective probability elicitation was a promising method to obtain the required conditional probabilities for data conditions that would be typical of small/rural systems. Three experts were identified: Expert A, Manager - Construction and Maintenance Engineering; Expert B, Director - District Operations; and Expert C, District Supervisor - Regional Operations. Three experts were sought because it is considered an appropriate number for group assessments (O'Leary 1998, Clemen and Winkler 1999, Renooij 2001). It is also unlikely that a small WDS would have more than three available experts. The three experts were involved with the construction of the case study system, its day to day operations, or both.

A questionnaire containing 43 questions was developed to solicit probabilities from the experts; a sample question of which is shown in Table 1. The questions ask directly for prior and conditional probabilities or they ask for a frequency assessment of certain events. The frequency approach was used where possible because it related most directly to the experience of the experts. For the other questions, especially those with many conditions, a probability scale was used to assist experts in determining the probabilities of the events they were considering. For each "total chlorine residual" and "pressure" measurement node, probability assessments were made assuming current monitoring conditions and extensive real-time monitoring. 
Table 1. Question eliciting the probability of contamination in pipe sections $\mathrm{P} 1$ and $\mathrm{P} 3$

\begin{tabular}{|c|c|c|c|}
\hline State of Nature & \multirow{2}{*}{ Intrusion P1 P3 } & Contamination from Inlet & Probability \\
\hline \multirow{3}{*}{$\begin{array}{c}\text { CP1-P3 }= \\
\text { TRUE }\end{array}$} & TRUE & TRUE & \\
\cline { 2 - 4 } & FALSE & TRUE & \\
\cline { 2 - 4 } & TRUE & FALSE & \\
\cline { 2 - 4 } & FALSE & FALSE & \\
\hline
\end{tabular}

A possible bias introduced by the probability scale is the use of $1 \%$ to describe all low probabilities, since it was the lowest value on the scale and associated with the descriptor "impossible." The probability scale, adapted from Tang and McCabe (2007), is shown in Table 2.

Table 2. Probability scale used for elicitation

\begin{tabular}{|c|c|c|c|c|c|c|c|c|c|c|}
\hline Impossible & \multicolumn{2}{|c|}{ Improbable } & \multicolumn{2}{|c|}{ Uncertain } & $\mathbf{5 0 - 5 0}$ & \multicolumn{2}{|c|}{ Somewhat Expected } & \multicolumn{2}{c|}{ Probable } & Certain \\
\hline $1 \%$ & $10 \%$ & $20 \%$ & $30 \%$ & $40 \%$ & $50 \%$ & $60 \%$ & $70 \%$ & $80 \%$ & $90 \%$ & $99 \%$ \\
\hline
\end{tabular}

Elicitation took place in February 2007 in the City of Saskatoon, Saskatchewan, Canada. The training of the experts and the probability elicitation occurred in one work day since the experts were not all based in the same location. Initially, the experts were given a presentation on probability theory, Bayesian Belief Network theory and on approaches to the generation of probabilities. Experts were also made aware of heuristic traps that could bias their responses such as representativeness, anchoring, base-rate neglect and control (Renooij 2001). As an example, adjusting the probability of contamination at the inlet to determine the probability at a point downstream would be anchoring. During training, experts were asked to respond to probability questions to demonstrate their understanding.

The experts answered the first 8 questions together to gain familiarity with the exercise; the principal author was available to answer questions and to receive comments on the structure of the BBN. The remaining questions were answered without collaboration, which is preferred to maintain heterogeneity in the probability responses (Clemen and Winkler 1999).

The experts were all engineers and felt most comfortable trying to establish a quantitative basis for their probability estimates. For example, to determine the probability of "vulnerability" to intrusion, Expert A calculated the ratio of manholes/valve chambers and joints, to straight pipe for each pipe section. Similarly, the probability of an adverse pressure event was generated by approximating the number of hours per year that the system experienced extreme pressure transients; this value was converted to probability per day.

After completion and submission of the initial questionnaire, experts were given a second questionnaire that asked for the complement of the probabilities sought in the original. This follows from basic axiom that $P(A)+P(\neg A)=1$. For example, if in the first round they were asked the probability that $A=$ true given some evidence, they were asked the probability that $A=$ false given the same evidence in the second round. This was a quality control exercise to ensure that experts correctly understood the elicitation exercise and the rules of probability theory. The authors selected a tolerance of $\pm 10 \%$ as an acceptable deviation; it was hypothesised that a decision maker would be unlikely to change his or her conclusions for a variation of $10 \%$ or less. Only one of the experts performed satisfactorily on the exercise; i.e., provided estimates that were approximately equal to one minus their original answer. The other experts gave the same answer to the complementary question as they did to the original question resulting in contradictory information 
about the behaviour of the system. When questioned about their responses, the experts said that they had not read the question properly; likely a result of the time constraint on the elicitation process.

One month later, the quality control questions and some of the original questions were readministered to the experts who did not perform well. The results were greatly improved and the responses to the quality control question were well within tolerance range. Some of the original questions were repeated, and one expert's new responses were almost identical to his original responses, showing excellent temporal consistency. Additional follow-up was also conducted for frequency data that varied significantly between the three experts to be considered reliable estimates; the values were reassessed.

\section{Evaluation of Individual Expert BBNs}

Once the design elements of the BBN were finalized and the probability assessments were obtained, the data were entered into a BBN sofware. MSBNx (Microsoft Bayesian Network Editor and Tool kit) was selected because it performed the needed calculations and interfaced well with other Microsoft software such as Excel which was used for data entry.

Two BBNs were created for each expert; one using their probability assessments for actual monitoring conditions and the other for real-time monitoring. The models were then tested by instantiating (setting the state of) input nodes and observing the output of the seventeen hypothesis nodes.

Each model has tendencies reflecting the understanding and assumptions of the expert providing the probability relationships. For example, the probability of intrusion in Expert A's BBN remains consistently lower than the other experts' BBNs even when the probability of adverse pressure is high; this may reflect his belief in the integrity of the system and in the lack of severity of adverse pressure events. Expert B's BBN ignores the effect of water temperature on chlorine residual and interprets every low chlorine event as a high probability of contamination. Expert C's BBN yields a high probability for the alternative causes of low chlorine residual measurements and only predicts contamination when these have been ruled out.

This step indicated that none of the individual expert BBNs yielded illogical outcomes and points to the influences of each expert data set in an integrated BBN.

\section{Sensitivity to Evidence}

The sensitivity of each expert's BBN to different evidence was evaluated using statistical entropy. Node instantiations that reduce uncertainty to the greatest extent represent valuable information that would be worth acquiring. In the context of the case study BBN, if an additional sensor significantly reduced the level of uncertainty about the contamination of an area, then its purchase and installation would be justified.

Mathematically, statistical entropy change quantifies the degree to which the probability mass is scattered among the node states and the extent to which evidence would cause the probability to approach either 0 or 1 (Jensen 1996). The entropy expression is given by Equation 6 (Jensen 1996):

$$
\operatorname{ENT}(P(H))=\sum_{i=1}^{n} P\left(h_{i}\right) \log _{2} P\left(h_{i}\right)
$$

where $H=$ node of interest, $h_{i}=$ all possible states, and $i=1, \ldots, n$ are the possible states of node $H$. The impact of evidence on the entropy of a hypothesis node is assessed by calculating the resulting change in entropy. Equation 7 incorporates this additional step (Jensen 1996): 


$$
\operatorname{ENT}(P(H))-E N T\left(P\langle H \mid E\rangle=\sum_{i=0}^{n} \sum_{j=0}^{n} P\left(h_{i}, e_{j}\right) \log _{2} P\left\langle h_{i} \mid e_{j}\right\rangle\right.
$$

where $\mathrm{H}=$ node of interest, $\mathrm{E}=$ evidence node whose state is varied, $\mathrm{h}_{\mathrm{i}}=$ all possible states, $\mathrm{i}=$ $1, \ldots, \mathrm{n}$ are the possible states of node $\mathrm{H}$ and the $\mathrm{e}_{\mathrm{j}}=$ all possible states of the evidence node $\mathrm{E}$. The analysis of network sensitivity to evidence was performed with Netica, ${ }^{\mathrm{TM}}$ a belief network software with a function to calculate sensitivity to evidence as MSBNx could not have been used to perform this analysis without additional programming. The Netica ${ }^{\mathrm{TM}}$ 'Sensitivity to Findings' function was run for the same sixteen hypothesis nodes.

The "sensitivity to findings" analysis did not conclusively demonstrate the need for realtime monitoring as a means to improve system water quality detection performance. Instead it revealed that experts over-estimated the probability of current daily monitoring practices detecting water quality failure. The entropy change with current sampling practices was almost identical to entropy results using the probability assessments for the hypothetical real-time scenario; the exception by a small margin was Expert $\mathrm{C}$. The results also indicate that current water quality monitoring at the inlet did not affect uncertainty relating to downstream water quality nodes. They suggest that additional daily or real-time water quality sampling at even one downstream point would reduce uncertainty for the rest of the system nodes by approximately $25-40 \%$. Finally, the analysis demonstrated the sensitivity of key hypothesis nodes, those representing the probability of contamination from an area, to each other.

A limitation of the statistical entropy analysis is that only one node is instantiated at a time. Possible combinations of nodes that could result in a significant reduction in uncertainty were not identified.

\section{Sensitivity to Probabilities}

To account for the impact of errors in the probability estimates of the experts, a one-way sensitivity-to-probabilities analysis was conducted for the probability tables of each expert. To perform this analysis, the probability table values of all child nodes in the network were varied three ways and the resulting changes in the probabilities of hypothesis nodes were documented. For sixteen sets of hypothetical observed states, each probability was increased and decreased by approximately $20 \%$ and varied again by approximately $5 \%$. Probabilities representing rare events (less than $1 \%$ probability) were varied by orders of magnitude $(10 \%, 1 \%, 0.1 \%, 0.01 \%, 0.001 \%)$ depending on their initial value. In general, they were increased once and decreased twice.

During elicitation, experts found it difficult to describe the low probability events, and the use of a probability scale with a lower bound of $1 \%$ added to this challenge. Experts often described low probability values as less than $1 \%$ or less than $0.1 \%$. Since a value had to be chosen for the model, "less than" was interpreted as a decrease by an order of magnitude; for example $<1 \%$ became $0.1 \%$. This rule was considered conservative and appropriate in that, it is a major change and would yield a significant sensitivity response if accuracy in the assessment of low probabilities was important to overall BBN behavior and the experts' decision making.

These tests indicated that changes in the results of the hypothesis nodes were less than $50 \%$ of the change in the probability table value, which was the decision rule used to determine acceptability. However, there were important exceptions to this, notably the variation of table values that represent "false" conditions; i.e., the system being in a given state in the absence of precursor or causal conditions. Errors in these probabilities could represent a different understanding of the system or indicate that an additional parent node is needed. The best way to address this is through detailed study of the behaviour of the WDS and of the performance of 
instruments used in the system. The sensitivity results also indicate that hypothesis nodes are most sensitive to changes in the probability of false positives of water quality measurement nodes; this was expected. Additional data regarding the relationship between sensor response, actual contamination and potential contamination causing events (intrusions) is needed in order to address this issue and provide more confidence in the probabilities and in the output of the BBN.

The assumption used to interpret the "less than" sign was shown to be appropriate because decreasing rare-event probabilities resulted in changes elsewhere of less than 5\%. A BBN user would be unlikely to change his or her decision based on such a small change.

\section{Combining Expert Probability Data}

Approaches for combining the knowledge of multiple experts into single probability values for use in one BBN include behavioural and mathematical methods (Clemen and Winkler 1999). The latter includes averaging, weighting, and linear and logarithmic opinion pools (Tang 2005). A Bayesian updating technique was selected for this methodology because it addressed two important considerations: benefiting from the heterogeneity of expert knowledge and managing the quality of expert judgements especially from small groups in which overconfidence, in particular, could have a significant impact (Clemen and Winkler 1999). Behavioral methods were not used to avoid any dominance experts within such a small group.

In the Bayesian approach (Stiber et al 2004), posterior probabilities are calculated for hypothesis nodes in each expert model and these are then weighted based on the posterior probability that an expert is correct, given a set of observations. Equations 8-11 (Stiber et al. 2004) are used in this method:

$$
P(\theta)=\sum_{i=1}^{n} P\left\langle\theta \mid M_{i}\right\rangle \times P\left(M_{i}\right)
$$

where $M_{i}=$ model elicited from expert i, $\theta=$ event of interest, $\mathrm{n}=$ number of experts, $P\left(M_{i}\right)$ $=$ probability that expert model $\mathrm{i}$ is correct and $P\left\langle\theta \mid M_{i}\right\rangle=$ probability of $\theta$ given that expert model $\mathrm{i}$ is correct, and

$$
P\left\langle M_{i} \mid x\right\rangle=\frac{P\left\langle x \mid M_{i}\right\rangle \times P\left(M_{i}\right)}{\sum_{j=1}^{n} P\left\langle x \mid M_{j}\right\rangle \times P\left(M_{j}\right)}
$$

where $\mathrm{x}$ is equal to observed evidence. The term $P\left\langle\theta \mid M_{i}\right\rangle$ in Equation 8 is obtained from each expert model once evidence has been entered. The term $P\left(M_{i}\right)$ in Equation 8 is equal to $P\left\langle M_{i} \mid x\right\rangle$ for each expert $\mathrm{i}$, which is obtained from Equation 9. A final assumption is that the expert models are mutually exclusive and exhaust the event space under consideration; therefore, the following relationship holds:

$$
\sum_{i=1}^{n} P\left(M_{i}\right)=1.0
$$

Finally, Equation 11 provides the probability of a given combination of evidence occurring in each expert model, where evidence $\mathrm{x}$ is composed of $\mathrm{K}$ individual observations $\left(\mathrm{e}_{1}, \ldots, \mathrm{e}_{\mathrm{k}}\right)$ for model nodes $\left(E_{1}, \ldots, E_{k}\right)$ :

$$
P\left\langle x \mid M_{i}\right\rangle=P_{i}\left(E_{1}=e_{1}\right) \times P_{i}\left(\left\langle E_{2}=e_{2} \mid E_{1}=e_{1}\right\rangle\right) \times \prod_{k=3}^{K} P\left(\left\langle E_{k}=e_{k} \mid\left(E_{1}=e_{1}\right) \cap \ldots \cap\left(E_{k-1}=e_{k-1}\right)\right\rangle\right)
$$


To perform these operations, a program named Integrated Expert BBN was written in Microsoft Visual Basic. ${ }^{\mathrm{TM}}$ The program is designed to collect data on the state of input nodes and send the evidence to each MSBNx expert model. The output probabilities are calculated in each model and returned to calculate a single probability for each hypothesis node.

Table 3 presents examples of inference results and weightings for two different sets of observations.

Table 3. Sample of integrated expert output and expert weighting

\begin{tabular}{|c|c|c|c|c|c|c|c|c|}
\cline { 2 - 10 } \multicolumn{1}{c|}{} & \multicolumn{2}{c|}{ Expert A } & \multicolumn{2}{c|}{ Expert B } & \multicolumn{2}{c|}{ Expert C } & \multicolumn{2}{c|}{ Integrated Probability } \\
\hline Hypothesis Node & Case 1 & Case 2 & Case 1 & Case 2 & Case 1 & Case 2 & Case 1 & Case 2 \\
\hline Adverse Pressure P1P3 & 0.00 & 0.93 & 0.00 & 0.74 & 0.00 & 0.52 & 0.00 & 0.53 \\
\hline Adverse Pressure P2P4P6 & 0.01 & 0.01 & 0.01 & 0.01 & 0.01 & 0.01 & 0.01 & 0.01 \\
\hline Adverse Pressure P5P7 & 0.01 & 0.00 & 0.01 & 0.01 & 0.01 & 0.01 & 0.01 & 0.01 \\
\hline Contamination Source & 0.60 & 0.00 & 0.96 & 0.00 & 0.36 & 0.00 & 0.58 & 0.00 \\
\hline Contamination P1P3 & 0.74 & 0.15 & 1.00 & 0.15 & 0.46 & 0.04 & 0.68 & 0.04 \\
\hline Contamination P2P4P6 & 0.66 & 0.26 & 0.99 & 1.00 & 0.45 & 0.04 & 0.65 & 0.05 \\
\hline Contamination P5P7 & 0.60 & 0.27 & 0.98 & 1.00 & 0.44 & 0.04 & 0.62 & 0.05 \\
\hline Intrusion P1P3 & 0.00 & 0.03 & 0.00 & 0.07 & 0.01 & 0.08 & 0.01 & 0.08 \\
\hline Intrusion P2P4P6 & 0.00 & 0.02 & 0.00 & 0.04 & 0.00 & 0.00 & 0.00 & 0.00 \\
\hline Intrusion P5P7 & 0.00 & 0.00 & 0.00 & 0.00 & 0.00 & 0.00 & 0.00 & 0.00 \\
\hline Weights & $\mathbf{0 . 3 1}$ & $\mathbf{0 . 0 2}$ & $\mathbf{0 . 2 5}$ & $\mathbf{0 . 0 0}$ & $\mathbf{0 . 4 4}$ & $\mathbf{0 . 9 8}$ & $\mathbf{1}$ & $\mathbf{1}$ \\
\hline
\end{tabular}

\section{Expert Model Weighting}

Testing was conducted on individual expert BBNs and on the Integrated Expert BBN to verify the behaviour of each. From initial tests using 18 sets of observations, it was shown that Expert $\mathrm{C}$ was frequently assigned a higher weight than either Expert $\mathrm{A}$ or $\mathrm{B}$. An evaluation of $\mathrm{BBN}$ weighting during the performance analysis step of this research confirmed the dominance of Expert C's model, particularly when there was deviation from typical operating conditions. This was not unexpected as Expert $\mathrm{C}$ works the most with the system and may have a more refined understanding of possible system behaviours.

\section{BBN Performance}

The combined expert BBN was tested using historical data comprising over 1600 time intervals of chlorine residual concentration and pressure measurements from the North Booster Pumping Station and daily chlorine and pressure measurements from the five customer towns. These data included a pipe break, a water quality failure lasting 22 hours and an operational change. Certain outcomes of this performance analysis are given here, but the details can be found in Joseph (2007).

Overall, the BBN tends to direct the attention of the user to other causes of adverse sensor readings that have a higher probability of occurrence, such as an operational change, so that they can be ruled out. It also correctly interpreted most adverse readings in the dataset. During the 22hour chlorine residual failure, the probability of downstream contamination in all pipe areas increases significantly, as expected. The system interprets the source of the problem as Contamination from the City of Saskatoon whose probability increased by four orders of magnitude from $5 \times 10^{-4} \%$ to $4 \%$, similar to an increase observed in Dawsey (2006). For this same event, the probability of Exposure Risk 1 exceeds the probability of Intrusion P1P3, which does 
not occur under normal operating conditions, indicating the alternative cause of the health exposure risk.

Another preliminary finding from the performance analysis is that the model currently does not take sufficient advantage of the availability of time related data, which would be abundant in the real time context and could reduce uncertainty about the reliability of sensor data. Also, the current node state definitions for Adverse Pressure are not sufficiently refined to distinguish between pressure loss and a pressure transient. Consequently, the BBN assigned the same probabilities of Adverse Pressure, Intrusion and Contamination to the pipe break event and the operational change event, both of which had resulted in chlorine residual measurements of $0 \mathrm{mg} / \mathrm{L}$. This must be corrected to improve the validity of the current model.

\section{Conclusions}

The method used to develop the present BBN involves the elicitation and combining of probabilities from multiple experts in support of the use of real-time monitoring for water quality regulatory compliance. The analysis of the method has revealed several insights and options for improved outcomes.

The critical element in this methodology involves the use of experts in the generation of probabilities for various system conditions for which data is not available. In the context of small WDSs, it is likely that the number of experts for a given system would be small and that, in a given group, one expert may know the system better than others. This was true for the case study system. A valuable intermediate step in the elicitation process would have been to conduct a behavioural integration using a Delphi method (Clemen and Winkler 1999). It would have allowed the slightly less familiar experts, who were also less involved in the BBN structure development, an opportunity to consider their responses in light of the anonymous assessments of their colleagues.

The two sensitivity analyses identified data collection needs and refinement priorities for probability assessment. The sensitivity analysis of the BBN probability tables demonstrates that the probabilities associated with false conditions require special attention, as their variation results in the most significant output changes. Additional effort could then be invested in the generation of these probabilities. Detailed study of the WDS and of the reliability of real-time monitoring equipment are examples of steps to be taken to obtain necessary data for probability refinement.

Finally, the performance evaluation of the BBN yielded several promising results notably its accurate interpretation of various system events in the historical dataset. However, refinements in the node structure to transmit data over time and to improve the node state definition of Adverse Pressure could yield valuable performance improvements. These changes may result in a node structure that requires the assessment of more than 8 probabilities for any given node. However, this could be overcome through decomposition methods and would reduce the number of probabilities to be considered simultaneously, thus improving an expert's precision in assessing them (Keeney and Von Winterfeldt 1991, Tang 2005).

A validation step to compare sensor performance and BBN output with actual water quality analyses taken at a suitable frequency is recommended for future research. Additional research aimed at improving remote monitoring tools for water quality especially with respect to intrusion events would also be beneficial.

In a scaling up of this methodology to a larger system BBN, developers would likely benefit from additional experts, hydraulic models and historical data with greater spatial and temporal sampling density. This would facilitate model structure development, probability elicitation and performance testing. Potential challenges include a greater number of parent nodes affecting each 
node, the impact of peak versus non-peak flow in terms of dependency relationships in the network and the ability of experts to assess probabilities given the more complex behaviour. Future research should explore the ability of expert to elicit probabilities for BBNs of larger systems such that outputs correspond acceptably to the results of a validation.

\section{Acknowledgements}

The authors are grateful for the contributions of Dr. Bryan Karney in the development of this methodology. We would also like to thank the staff at SaskWater Inc. as well as that of the representatives from the towns of Martensville, Dalmeny, Osler and Hague, Saskatchewan for their collaboration. Special thanks go to B.S. Jong for his preliminary research and development of Figure 1. Finally, the present work would not have been possible without the financial support of the Canadian Water Network and the National Science and Engineering Research Council (NSERC).

\section{References}

Bristow, E. and Brumbelow, K. (2006) "Delay between Sensing and Response in Water Contamination Events," Journal of Infrastructure Systems, ASCE, 12(2), 87-95

Clemen, R. T. and Winkler, R.L. (1999) "Combining Probability Distributions from Experts in Risk Analysis," Risk Analysis, 19 (2), 187-203

Coupé, V. M. H. and Van Der Gaag, L. C. (2002) "Properties of Sensitivity Analysis of Bayesian Belief Networks," Annals of Mathematics and Artificial Intelligence, 32(4), 232-256.

Dawsey, W. J., Minsker, B. S. and Van Blaricum, V. L. (2006) "Bayesian Belief Networks to Integrate Monitoring Evidence of Water Distribution System Contamination," Journal of Water Resources Planning and Management, ASCE, 132(4), 234-241.

Hrudley, S. and Hrudley, E. (2004) Safe Drinking Water - Lessons from Recent Outbreaks in Affluent Nations, , IWA Publishing, Cornwall, UK.

Jensen, F. V. (1996) An Introduction to Bayesian Networks, Springer-Verlag, New York, NY.

Joseph, S. (2007) The Effectiveness of Bayesian Belief Networks and Real-Time Monitoring Technology in Facilitating the Implementation of Water Quality Regulations. M.A.Sc Thesis, University of Toronto, Toronto, ON.

Keeney, R.L. and Von Winterfeld, D. (1991) "Eliciting Probabilities from Experts in Complex Technical Problems," Transactions on Engineering Management, 38(3), 191-201

Lindley, T. \& Buchberger, S. (2002) Assessing Intrusion Susceptibility in Distribution Systems. Journal of the American Water Works Association, 94(6), 66-79.

McInnis, D. (2004) "A relative-risk framework for evaluating transient pathogen intrusion in distribution systems." Urban Water Journal, 1(2), 113-127.

Meyer, M.A., and Booker, J.M., (2001) Eliciting and Analyzing Expert Judgement: A practical guide, Academic Press Ltd., London

O'Leary, D. (1998) "Knowledge Acquisition from Multiple Experts: An Empirical Study," Management Science, 44, 1049-1058.

Pearl, J. (1988) Probabilistic Reasoning in Intelligent Systems: Networks of plausible inference, Morgan Kaufmann, San Francisco CA.

Renooij, S. (2001) "Probability Elicitation for Belief Networks: Issues to Consider," The Knowledge Engineering Review, 16(3), 255-269.

Robertson, W., Stanfield, G., Howard, G. \& Bartram, J. (2003) Chapter 6 - Monitoring the Quality of Drinking Water During Storage and Distribution. IN WHO (Ed.) Assessing 
Microbial Safety of Drinking Water, IWA Publishing, Cornwall, UK.

Stiber, N.A., Small, M.J., Pantazidou, M. (2004) "Site Specific Updating and Aggregation of Bayesian Belief Network Models for Multiple Experts," Risk Analysis, 24(6), 1529-1538

Strauss, J. (2005) "Escape from Kashechewan." Globe and Mail, Toronto, ON (28 October 2005).

Tang, Z. and McCabe, B. (2007) "Developing Complete Conditional Probability Tables from Fractional Data for Bayesian Belief Networks," Journal of Computing in Civil Engineering, ASCE, 21(4), 265 - 276

Tang, Z. (2005) Developing Conditional Probability Tables from Fractional Data for Bayesian Belief Networks in Engineering Decision Making, (Online) PhD thesis, University of Toronto, Toronto, ON. Available from: http://proquest.umi.com/pqdlink?did=1001862061\&Fmt=7\&clientId=12520\&RQT=309 $\&$ VName $=$ PQD (Accessed 11 September 2006).

Tversky, A., and Kahneman, D. (1974) "Judgements Under Uncertainty: Heuristics and Biases," Science, New Series, 185(4157), 1124-1131 